\title{
Solubilization of Dipropofol, an Antibacterial Agent, Using Saccharide and Ascorbic Acid
}

\author{
Masahiro OGata, ${ }^{*, a, c}$ Kentaro OKa, ${ }^{b}$ Masako Seki, ${ }^{b}$ Midori Hoshi ${ }^{b}$ Hirokatsu TAKatsu, ${ }^{c}$ \\ Tadahiko Mashino, ${ }^{b}$ Shiro Urano, ${ }^{c}$ and Toyoshige ENDO ${ }^{b}$ \\ ${ }^{a}$ Aomori University; 2-3-1 Kohbata, Aomori, Aomori 030-0943, Japan: ${ }^{b}$ Kyoritsu University of Pharmacy; 1-5-30 \\ Shiba-koen, Minato-ku, Tokyo 105-8512, Japan: and ' Shibaura Institute of Technology; 3-9-14 Shibaura, Minato-ku, \\ Tokyo 108-8548, Japan. Received November 8, 2006; accepted June 7, 2007; published online June 7, 2007
}

\begin{abstract}
Dipropofol has a strong antibacterial activity against Gram-positive bacteria. However, it lacked the solubility in water and this property was supposed to limit its efficacy. We tried to improve the solubility and found a new solubilization method of dipropofol in water by the addition of a monosaccharide or ascorbic acid.
\end{abstract}

Key words dipropofol; monosaccharide; ascorbic acid; solubilization

For more than 30 years, vancomycin has been a reliable treatment for Gram-positive bacterial infection. Injectable forms of vancomycin were introduced, and have been used exclusively for methicillin-resistant Staphylococcus aureus (MRSA) infection. Recently, the MRSA strain, which contains the vanA gene, with high resistance to vancomycin (MIC $>128 \mu \mathrm{g} / \mathrm{ml}$ ) has been reported in clinical isolates. ${ }^{1,2)}$ The emergence of vancomycin resistant bacterial strains is a very serious public health problem. Therefore, a new antiMRSA antibiotic is clinically of interest.

Dipropofol (Fig. 1) was recognized as an oxidative dimerization metabolite of propofol (2,6-diisopropylphenol), a novel sedative and anesthetic agent having antioxidant activity, but not antibacterial activity. ${ }^{3,4)}$ Dipropofol was investigated to have a higher antioxidant activity than that of propofol, and to show potent antibacterial activity against Grampositive strains including MRSA and vancomycin resistant Enterococci (VRE) ${ }^{4,5)}$

However, the insolubility of dipropofol in water could be plausible for its efficacy and application fields. Consequently, the solubilization of dipropofol in water was a next target. To obtain the water-solubility of the compounds, many researchers have used synthetic approaches and selected suitable derivatives. ${ }^{67)}$ However, we selected another method of adding other types of compounds, expecting complex formations and synergistic activity with the additions.

\section{MATERIALS AND METHODS}

Chemicals Dextrose anhydrous, saccharose, ascorbic acid and lecithin (from egg) were purchased from the Wako Pure Chemical Co. (Osaka, Japan). D(-)-Fructose, D (+)mannose, $\mathrm{D}(+)$-xylose, maltose monohydrate, and D-sorbitol were purchased from Sigma-Aldrich Japan K.K. (Tokyo, Japan). Dipropofol was synthesized by the method reported previously. ${ }^{4)}$

Antimicrobial Susceptibility The disk-diffusion test, using S. aureus 209P and E. coli JCM5491 (ATCC25922)

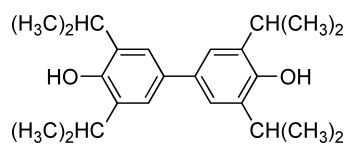

Fig. 1. Structure of Dipropofol was applied to determine the antimicrobial activity of the test compounds. The test organisms were grown overnight at $37^{\circ} \mathrm{C}$ by shaking in a TSB medium and the aliquots $(100 \mu \mathrm{l})$ were streaked onto trypticase soy agar plates. A dipropofol solution $(1 \mathrm{mg} / \mathrm{ml}$ in methanol) was suspended with saccharide $(1 \mathrm{~g})$ or ascorbic acid $(1 \mathrm{~g})$. The perfect removal of methanol produced a dipropofol complex with saccharide or ascorbic acid, which dissolved in distilled water $(5 \mathrm{ml})$. For plate diffusion tests, $50 \mu \mathrm{l}$ of the test compound was dropped on paper disks (diameter $8 \mathrm{~mm}$ ), which were dried under sterile conditions and put on and an agar plate inoculated with the test organism. The plates were cultivated at $37^{\circ} \mathrm{C}$ for $24 \mathrm{~h}$.

Combination Study and Synergism Determination The in vitro combinational effect of the dipropofol with ascorbic acid was examined by the checkerboard titration method. Overnight a culture of $S$. aureus, grown in $10 \mathrm{ml}$ of Mueller-Hinton broth at $37^{\circ} \mathrm{C}$, was diluted $10^{3}$-fold with fresh Mueller-Hinton broth, followed by the addition of $S$. aureus (about $5 \times 10^{4} \mathrm{CFU}$ ) onto $20 \mathrm{ml}$ Mueller-Hinton agar layers which contained dipropofol $(0-6.25 \mu \mathrm{g} / \mathrm{ml})$ and ascorbic acid $(0-12.5 \mathrm{mg} / \mathrm{ml})$. After $18 \mathrm{~h}$ of incubation at $37^{\circ} \mathrm{C}$, the MIC value was determined for each fraction. The fractional inhibitory concentration (FIC) for each component was calculated based on the following formula: FIC index $=(\mathrm{MIC}$ of drug $\mathrm{A}$, tested in combination $) /(\mathrm{MIC}$ of drug $\mathrm{A}$, tested alone $)+(\mathrm{MIC}$ of drug $\mathrm{B}$, tested in combination)/ (MIC of drug B, tested alone). The interaction was defined as synergistic if the FIC index was $<0.5$, additive if the FIC index was $>0.5$, and antagonistic if the FIC index was $>4 .{ }^{8}$ )

Oxidation Reduction Potential The oxidation reduction potential of dipropofol and ascorbic acid were estimated using cyclic voltammetry methodology. $\left.{ }^{9}\right)$ The cyclic voltammeter used for these studies was obtained from POTENTIOSTAT/GALVANOSTAT HA-301 (Hokuto Denko Co., Ltd., Japan). A three-electrode system was employed with $\mathrm{Ag} / \mathrm{AgCl}$ as the reference electrode, a glassy carbon electrode as the working electrode and a platinum wire as a counter electrode. The cell contains $0.5 \mathrm{~mm}$ sample and $N, N$ dimethylformamide (contain $60 \mathrm{~mm}$ tetra- $n$-butylammonium hexafluorophosphate): $41.75 \mathrm{~mm}$ MES buffer ( $\mathrm{pH}$ 7.4) $(=6: 4)$. The cyclic voltammetry tracings were recorded from $-0.2 \mathrm{~V}$ to $1.5 \mathrm{~V}$ at a scan rate of $50 \mathrm{mV} / \mathrm{s}$.

Synthesis of $3,3^{\prime}, 5,5^{\prime}$-Tetraisopropyldiphenoquinone 
(Dipropofolquinone) Propofol $(1 \mathrm{~g})$ was dissolved in $\mathrm{CH}_{2} \mathrm{Cl}_{2}(10 \mathrm{ml})$ and stirred with $\mathrm{CuCl}(\mathrm{OH}) \cdot$ tetramethylethylenediamin (TMEDA) $(16 \mathrm{mg})$ for $5 \mathrm{~h}$ at room temperature. The reaction product was extracted with AcOEt, after removal of the solvent, and crystallized from hexane to give the dipropofolquinone $(990 \mathrm{mg}, 99 \%)$ as reddish violet rods. FAB-MS $m / z: 353(\mathrm{M}+\mathrm{H})^{+} .{ }^{1} \mathrm{H}-\mathrm{MNR}\left(300 \mathrm{MHz}, \mathrm{CDCl}_{3}, \delta\right.$, ppm): $1.20\left(24 \mathrm{H}, \mathrm{d}, J=6.7 \mathrm{~Hz}, 8 \times \mathrm{CH}_{3}\right), 3.20(4 \mathrm{H}, \mathrm{m}$, $J=6.7 \mathrm{~Hz}, 4 \times \mathrm{CH}), 7.62\left(4 \mathrm{H}, \mathrm{s}, \mathrm{H}-2,2^{\prime}, 6,6^{\prime}\right) . \mathrm{mp} 225^{\circ} \mathrm{C}$.

Reduction of Dipropofolquinone with Ascorbic Acid or Saccharides Dipropofolquinone $(20 \mathrm{mg})$ was dissolved in ethanol $(99 \mathrm{ml})$ and stirred with ascorbic acid $(200 \mathrm{mg}$, $20 \mathrm{mg}$, or $2 \mathrm{mg}$ ) (dipropofolquinone: ascorbic acid=1:10, $1: 1,10: 1)$ for $4 \mathrm{~h}$ at room temperature. Glucose $(2 \mathrm{~g})$, fructose $(2 \mathrm{~g})$, mannose $(2 \mathrm{~g})$, xylose $(2 \mathrm{~g})$, or sorbitol $(2 \mathrm{~g})$ were dissolved in water $(1 \mathrm{ml})$ and stirred with dipropofolquinone in the same manner and the plausible reaction was monitored with TLC. The reaction product was extracted with AcOEt and the solvent was evaporated. The reactant was analyzed with TLC (hexane: AcOEt $=10: 1$ ) and ${ }^{1} \mathrm{H}-\mathrm{MNR}$.

Stability of Dipropofol Dipropofol $(10 \mathrm{mg})$ was dissolved in ethanol $(9 \mathrm{ml})$, added to water $(\mathrm{pH} 7,1 \mathrm{ml})$, water $(\mathrm{pH} 2,1 \mathrm{ml})$, or ascorbic acid $(10 \mathrm{mg})$, glucose $(10 \mathrm{mg})$, fructose $(10 \mathrm{mg})$, mannose $(10 \mathrm{mg})$, xylose $(10 \mathrm{mg})$ or sorbitol $(10 \mathrm{mg})$ were dissolved in water $(1 \mathrm{ml})$. Those mixture solutions were irradiated with UV-C $(254 \mathrm{~nm}, 4.9 \mathrm{~W})$ for $24 \mathrm{~h}$ at room temperature. The reaction product was extracted with AcOEt, after the removal of the solvent, the reactant was analyzed with TLC (hexane : AcOEt $=10: 1$ ) and ${ }^{1} \mathrm{H}-\mathrm{MNR}$.

\section{RESULTS AND DISCUSSIONS}

For the solubilization of dipropofol, several procedures were examined. However, the salt formation with alkaline metals was found to decrease its activity, and an addition of dipropofol solution $(1 \mathrm{mg} / \mathrm{ml}$ in methanol) into distilled water or saccharide solution caused the liberation of the dipropofol. But as shown in Fig. 2, the direct addition of saccharide powder to a dipropofol solution gave a complex including dipropofol and saccharide after the perfect removal of methanol which could dissolve in distilled water (the same procedures were used for glucose, sucrose, fructose, mannose, xylose, maltose, and sorbitol).

To determine the antibacterial activity of the complex solution, Staphylococcus aureus 209P, grown in a Trypticase soy (TS) broth at $37^{\circ} \mathrm{C}$ overnight, was applied to the surface of the TS agar dishes, and these dishes were incubated at $37^{\circ} \mathrm{C}$ overnight (Fig. 3A, (a) $15 \mu \mathrm{l}$ sample solution together with one drop each of a $50 \mu \mathrm{M}$ solution and $(\mathrm{b}-\mathrm{d})$ a paper disk impregnated with sample solutions). The antibacterial activity was evaluated from the diameters of the inhibition zone. As shown in Figs. 3A and B, when a dipropofol-monosaccharide (glucose, fructose, mannose, xylose and sorbitol) complex was directly applied onto the plates, a clear antibacterial activity was observed. The paper disk did not show any zonal inhibition, however, clear growth inhibition was also observed under the disk. This phenomenon might suggest the higher ability complex formation of saccharides, which also induced the lack of diffusivity of the dipropofol-saccharide complex. Disaccharides, such as sucrose and maltose, have no antibacterial activity (Figs. 3B (6), (7)).

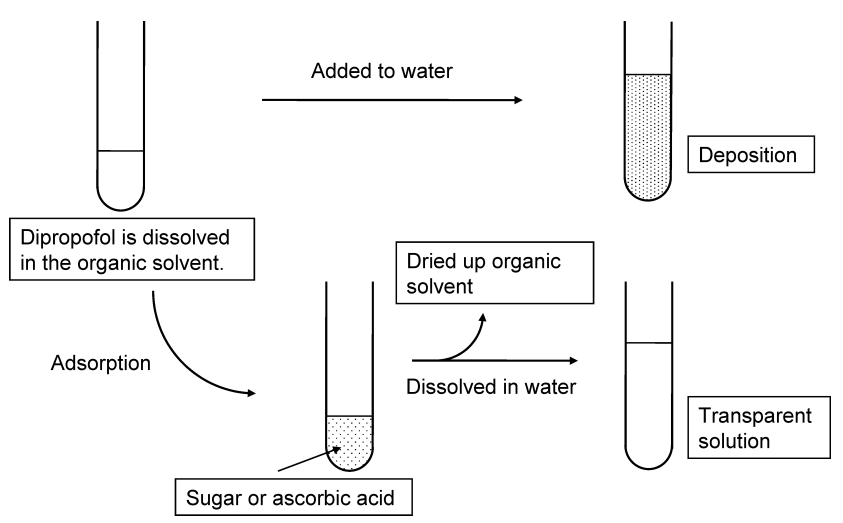

Fig. 2. An Outline of This Experiment

(A)

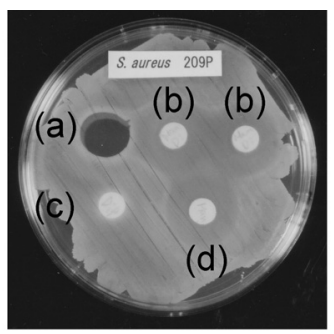

(B)

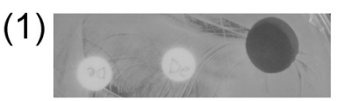

(5)

(2)

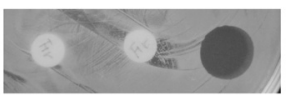

(3)

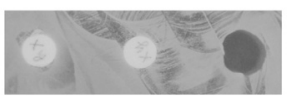

(6)

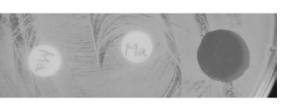

(4)

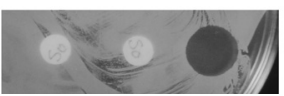

Fig. 3. The Antibacterial Activity of the Dipropofol Complex

(A) Dipropofol-mannose complex dissolved in water, (a) direct application of the complex solution, (b) the solution (a) was adsorbed on paper disks, (c) dipropofol was adsorbed on a paper disk from a methanol solution, or (d) mannose solution adsorbed on a paper disk. (B) Dipropofol complex with other saccharides, (1) glucose, (2) fructose, (3) xylose, (4) sorbitol, (5) mannose, (6) sucrose, or (7) maltose. Left and center were dipropofol-sugar complexes dissolved in water and adsorbed on paper disks. Right was the direct application complex of solution.

Also, when dipropofol is dissolved in a surface-active agent, such as Tween 20 and Tween 80, emulsion (including $1.2 \%$ lecithin and $10 \%$ soybean oil), which is utilized for the medical preparation of propofol and cyclodextrin, these solutions had no antibacterial activity (data not shown). These dipropofol complexes (disaccharides, surface-active agent, emulsion or cyclodextrin) were supposed to form micell, and hold the active site inside of the micell.

Another problem with decreasing the activity of the dipropofol was air oxidation to form dipropofolquinone, which has no antioxidant and antibacterial activities. It is well known that ascorbic acid is a kind of sugar and works as a reducing agent of $\alpha$-tocopherol in vivo, which is a lipophilic antioxidant. ${ }^{10)}$ Similarly, it could be expected to the show synergistic effect by the combined use of dipropofol and ascorbic acid.

As shown in Table 1, the minimum inhibitory concentrations (MICs) of dipropofol and ascorbic acid were 3.12 
$\mu \mathrm{g} / \mathrm{ml}$ and $6.25 \mathrm{mg} / \mathrm{ml}$ against $S$. aureus 209P, respectively. The MIC of dipropofol declined to $0.78 \mu \mathrm{g} / \mathrm{ml}$, when combined with ascorbic acid. A synergistic effect was obtained by the combined use of dipropofol and ascorbic acid. The data were analyzed using the fractional inhibitory concentration (FIC) index (FIC index was <0.5). The FIC index is the most frequently used method to determine the interactions between antimicrobial drugs. ${ }^{9)}$ The reactant of dipropofolquinone and ascorbic acid was analyzed using TLC and ${ }^{1} \mathrm{H}-$ NMR. The ${ }^{1} \mathrm{H}-\mathrm{NMR}$ identified the product of the $R f$ value 0.21 as dipropofol (data not shown) ${ }^{5)}$ and dipropofolquinone $(R f$ value 0.54$)$ was a result of recovering dipropofol $(R f$ value 0.21 ) with ascorbic acid (dipropofolquinone : ascorbic acid $=1: 10,1: 1$ ) (Fig. 4A-1). It could be reasonable to assume the ascorbate reduction action in relation with this synergism. On the other hand, the addition of saccharide did not stimulate the dipropofol formation reaction. The oxidation potential of dipropofol and ascorbic acid were $401 \mathrm{mV}$ and $303 \mathrm{mV}$, respectively, using cyclic voltammetry. This result is also supported by the ascorbate reduction action.

In addition, the stability of dipropofol was investigated by UV $(254 \mathrm{~nm})$ irradiation (Fig. 4A-2). In a solution containing ascorbic acid, the dipropofol was stable after $24 \mathrm{~h}$, but the solution of added water ( $\mathrm{pH} 2$ ) or glucose was not stable and dipropofolquinone was generated. We considered that the ascorbic acid worked as a reducing agent of dipropofol as is the case of $\alpha$-tocopherol and ascorbic acid (Fig. 4B).

In Fig. 5, a complex formation experiment was carried out using ascorbic acid instead of the saccharides. In this case of S. aureus 209P, a Gram-positive bacteria, zonal growth inhibition was observed by direct application as well as by the

Table 1. MICs $(\mu \mathrm{g} / \mathrm{ml})$ of Dipropofol and in Combination with Ascorbic Acid

\begin{tabular}{ccccccccccc}
\hline \hline & \multicolumn{10}{c}{$\mathrm{A} . \mathrm{A}(\mathrm{mg} / \mathrm{ml})$} \\
$(\mu \mathrm{g} / \mathrm{ml})$ & 12.5 & 6.25 & 3.12 & 1.56 & 0.78 & 0.39 & 0.19 & 0.09 & 0 \\
\cline { 2 - 9 } & & & - & - & - & - & - & - & - & - \\
\hline 6.25 & - & - & - & - & - & - & - & - & - \\
3.12 & - & - & - & - & - & - & - & + & + & + \\
1.56 & - & - & - & - & $-{ }^{a}$ & $\left.-{ }^{a}\right)$ & $\left.-{ }^{a}\right)$ & + & + & + \\
0.78 & - & - & - & + & + & + & + & + & + \\
0.39 & - & - & + & + & + & + & + & + \\
0.19 & - & - & + & + & + & + & + & + & + \\
0 & - & - & + & + & + & + & + & + & + \\
\hline
\end{tabular}

DP: dipropofol, A.A: ascorbic acid. + : growth, - : inhibition, a) synergism. paper disk applications, and their inhibition zones were larger than those of the ascorbic acid alone. This phenomenon showed a diffusible property of dipropofol into water in the presence of ascorbic acid. Moreover, the dipropofolascorbic acid complex inhibited the growth of $E$. coli JMC5491, a Gram-negative bacteria of less susceptible to many antibiotics and insensitive to dipropofol by their cell surface structure (Fig. 5, right). Growth inhibition was observed by direct application onto the plates, but it was also observed under the paper disk (data not shown).

Combinational administration could be an alternative to the development of new classes of agents ${ }^{11-14)}$ and we have studied the combined effect between dipropofol and other classes of antibiotics. The synergy against VRE was only

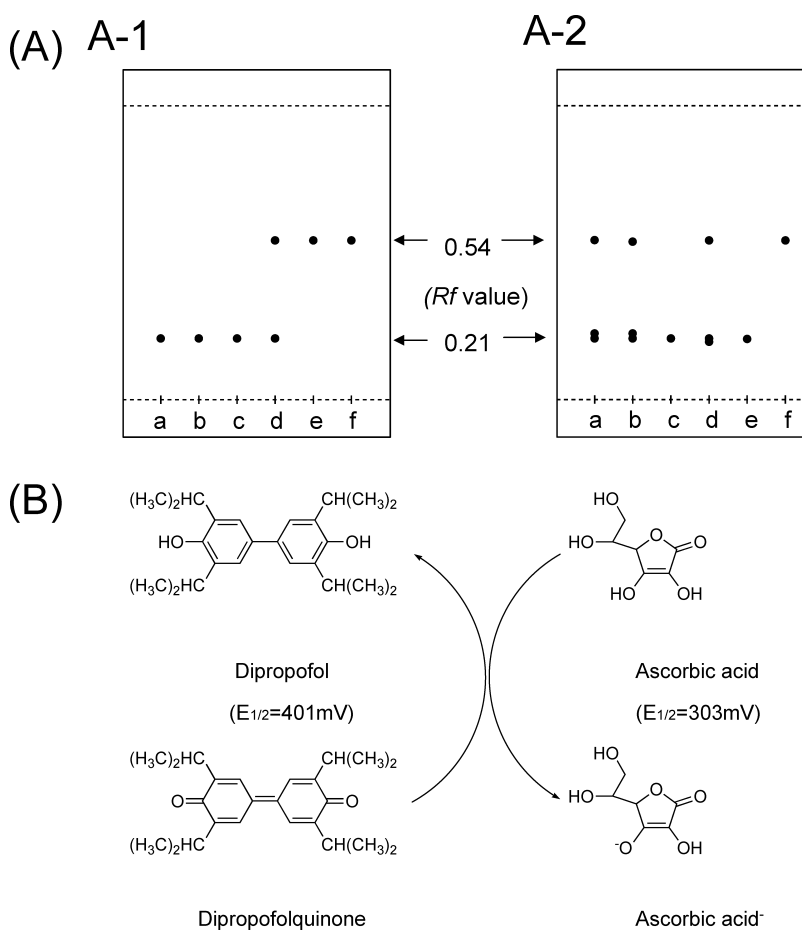

Fig. 4. Antibacterial Activity of the Dipropofol-Ascorbic Acid Complex

(A-1) Dipropofolquinone reductive reaction with ascorbic acid or sugar, a: dipropofol, b: dipropofolquinone : ascorbic acid $(1: 10)$, c: dipropofolquinone : ascorbic acid $(1: 1)$, d: dipropofolquinone: ascorbic acid $(10: 1)$, e: dipropofolquinone: glucose (1:100), f: dipropofolquinone. (A-2) Stability of dipropofol. a: dipropofol only, b: dipropofol+water $(\mathrm{pH} 2)$, c: dipropofol+ascorbic acid, d: dipropofol+glucose, e: dipropofol (not irradiated), f: dipropofolquinone (not irradiated). (B) The scheme of the oxidation-reduction reaction of dipropofol and ascorbic acid.
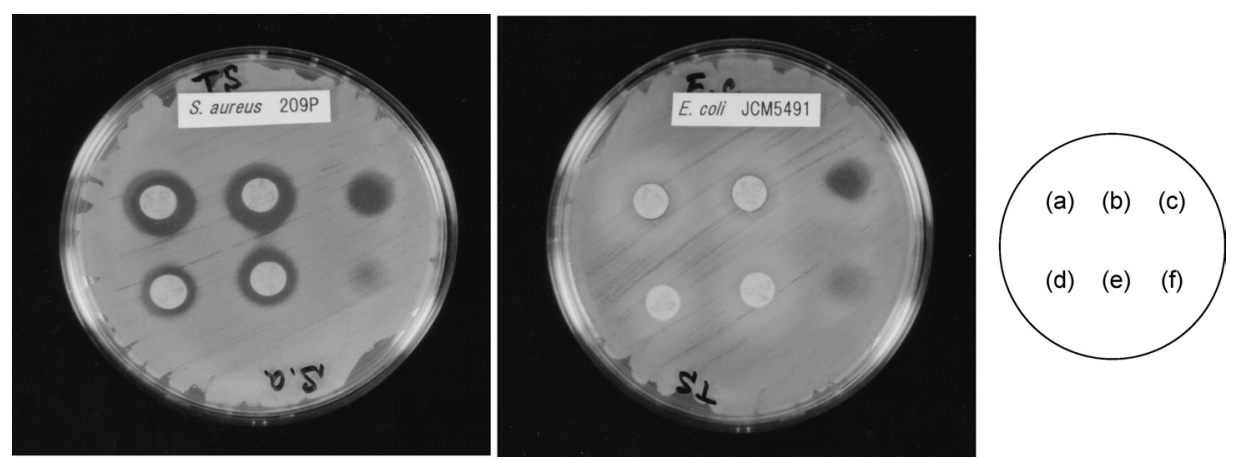

Fig. 5. Dipropofol-Ascorbic Acid Solution in Water, Left; S. aureus 209P, Right; E. coli JCM5491

(a) and (b) were paper disk with dipropofol-ascorbic acid complex solutions, (c) direct application of the dipropofol-ascorbic acid complex solution, (d) and (e) were paper disks with ascorbic acid alone, and (f) direct application of ascorbic acid alone in the medium. 
confirmed with the combination between dipropofol and rifampicin. ${ }^{15)}$ To our knowledge, this is the first study describing the enhancing activity of ascorbic acid to the antimicrobial compound, and in the presence of ascorbic acid dipropofol was solubilized in water, stable to air oxidation and showed synergistic potency against Gram-positive and Gramnegative bacteria. In conclusion, we could offer a new route for the development of solubilzing lipophilic compounds, which was abandoned due to strong lipohilicity. Detailed studies of the antibacterial activity in vivo are now under investigation.

Acknowledgments We thank Miss Masako Hirano and Mr. Yosuke Tosa for their helpful assistance. This work was supported in part by a research grant from the KANPO Science Foundation, Tokyo, Japan.

\section{REFERENCES}

1) Centers for Disease Control and Prevention, $M M W R$, 51, 565-567 (2002).

2) Centers for Disease Control and Prevention, $M M W R$, 51, 902 (2002).
3) Kvam C., Granese D., Flaibani A., Pollesello P., Paolletti S., Biochem. Biophys. Res. Commum., 193, 927-933 (1993).

4) Ogata M., Sato T. S., Kunikane T., Oka K., Seki M., Urano S., Hiramatsu K., Endo T., Biol. Pharm. Bull., 28, 1120-1122 (2005).

5) Ogata M., Shin-ya K., Urano S., Endo T., Chem. Pharm. Bull., 53, 344-346 (2005)

6) Yoshida O., Yasukata T., Sumino Y., Munekage T., Narukawa Y., Nishitani Y., Bioorg. Med. Chem. Lett., 12, 3027-3031 (2002).

7) Rubenstein M. S., Baichwal V., Beckmann H., Clark D. L., Frankmoelle W., Roche D., Santha E., Schwender S., Thoolen M., Ye Q. J., Jaen J. C., J. Med. Chem., 44, 3599-3605 (2001).

8) Bouanchaud H. D., J. Antimicrob. Chemother, 30 (Suppl. A), 95-99 (1992).

9) Chevion S., Roberts M., Chevion M., Free Radic. Biol. Med., 47, 1019-1027 (2000)

10) Sies H., Murphy M. E., J. Photochem. Photobiol. B, 8, 211-218 (1991).

11) Zhao W. H., Hu Z. Q., Okubo S., Hara Y., Shimaura T., Antimicrob. Agents Chemother. 45, 1737-1742 (2001).

12) Rand K. H., Houck H. J., Antimicrob. Agents Chemother, 48, 28712875 (2004).

13) Deshpande L. M., Jones R. N., Clin. Microbial. Infect., 9, 1120-1124 (2003).

14) Walsh C., Nature Rev., 1, 67-70 (2003).

15) Ogata M., Kunikane T., Seki M., Oka K., Urano S., Seki S., Seki Y., Endo T., Biol. Pharm. Bull., 28, 1773-1775 (2005). 